7-20-1985

\title{
Wave refraction by warm core rings
}

George R. Mapp

Virginia Institute of Marine Science

Christopher S. Welch

Virginia Institute of Marine Science

John C. Munday Jr.

Virginia Institute of Marine Science

Follow this and additional works at: https://scholarworks.wm.edu/vimsarticles

Part of the Marine Biology Commons

\section{Recommended Citation}

Mapp, George R.; Welch, Christopher S.; and Munday, John C. Jr., "Wave refraction by warm core rings" (1985). VIMS Articles. 295.

https://scholarworks.wm.edu/vimsarticles/295

This Article is brought to you for free and open access by W\&M ScholarWorks. It has been accepted for inclusion in VIMS Articles by an authorized administrator of W\&M ScholarWorks. For more information, please contact scholarworks@wm.edu. 


\title{
Wave Refraction by Warm Core Rings
}

\author{
George R. Mapp, ${ }^{1}$ Christopher S. Welch, and John C. Munday \\ Virginia Institute of Marine Science, School of Marine Science \\ College of William and Mary, Gloucester Point, Virginia
}

\begin{abstract}
A numerical model for refraction of ocean swell by currents associated with a warm core ring was developed and tested with Seasat synthetic aperture radar (SAR) data. The wave field of SAR orbit 1232 was measured using optical Fourier transforms. The wave refraction model produced rays by simultaneous, numerical integration of the Hamiltonian ray equations applied to a moving medium. Wave orthogonals were constructed from wave number vectors calculated at each incremental time step. The flow field used by the model to simulate a warm ring was a steady, circular jet, with the radial profile of tangential velocity composed of a power function joined to a Gaussian. Initial wave conditions for simulation of refraction by the SAR-imaged ring were determined from measurements outside the ring. No data were available from which to determine the current field of the SAR-imaged ring, so the current field input to the model was adjusted until the output wave field most nearly resembled the SAR observations. The relative locations of convergence and divergence of rays were as observed on the SAR image, and the relative energy density in crossed seas was correctly predicted. However, predicted patterns of wavelength variation (presuming that incident waves were uniform in wavelength) were not observed.
\end{abstract}

\section{INTRODUCTION}

During the summer of 1978 , several images of a ground swath extending from Cape Cod to Cape Hatteras were recorded by the Seasat synthetic aperture radar (SAR). In this region, warm core rings are often found as they migrate southward toward Cape Hatteras. One ring, labeled eddy "S" on NAVOCEANO frontal analysis maps, was identified by Lichy et al. [1981] on six SAR images by lighter tones and arcuate linear features suggestive of current shear. Two of these images were selected by the Jet Propulsion Laboratory (JPL) for digital processing to a resolution of $25 \mathrm{~m}$. Ocean swell appears throughout the digital image of orbit 1232, making it suitable for a study of refraction by surface currents. (See Vesecky and Stewart [1982, p. 3422] or Beal et al. [1981, p. 138] for a reproduction of this image.)

In the present study, a model was developed for wave refraction induced by currents associated with warm core rings. Wave conditions measured from the SAR imagery outside the ring were used to initialize the model, which constructs rays and orthogonals by simultaneously integrating the ray equations for a moving medium. The simulated wave field was then compared to the wave field in and beyond the ring, measured from the SAR imagery.

In the sections which follow, model development will first be explained. Then the process of measuring the wave field from imagery is described, and finally, predicted and observed wave fields are compared.

\section{Current-Induced Wave Refraction}

In the following discussion, rays are "paths traced out by points which move with the group velocity" [Kenyon, 1971] while orthogonals are lines which are everywhere perpendicular to wave crests. Refraction of orthogonals by a onedimensional shear current in deep water was first analyzed quantitatively by Johnson [1947]. He expressed Snell's law in the form

\footnotetext{
${ }^{1}$ Now at Computer Sciences Corporation, National Space Technology Laboratory, Mississippi.

Copyright 1985 by the American Geophysical Union.
}

$$
\frac{\sin \phi_{0}}{\sin \phi}=\left(1-\frac{U}{C_{0}} \sin \phi_{0}\right)^{2}
$$

where $\phi_{0}$ is angle of incidence, $\phi$ is angle of refraction, $U$ is current speed, and $C_{0}$ is initial phase speed.

Using (1), Hayes [1980] developed a model for currentinduced refraction of orthogonals, which was used to simulate refraction by the Gulf Stream near Marineland, Florida. The Gulf Stream was simulated by a varying, one-dimensional shear current, and the incident wave field was composed of initially parallel orthogonals.

In the case of a two-dimensional, spatially varying current (such as an eddy), the Hayes model is inappropriate. Instead, it is necessary to first calculate the trajectory of rays; then orthogonals can be interpolated from the wave number vectors calculated at points along each ray.

Refraction of rays by currents in deep water was discussed by Kenyon [1971]. A comprehensive discussion of the ray approach may be found in Leblond and Mysak [1978, pp. 24-28]. In this discussion, requirements are set for ray theory to be applicable. In our case, these requirements include that the wavelength of the swell be much less than the scale of current variability in the ring and that the time scale of ring velocity variation be much longer than the time for wave group passage through the ring. Assuming these requirements to be met, rays are calculated, given initial location and wave number vector, by simultaneously integrating the Hamiltonian ray equations for a moving medium:

$$
\begin{gathered}
\frac{d \mathbf{x}}{d t}=\frac{\partial \omega}{\partial \mathbf{K}} \\
\frac{d \mathbf{K}}{d t}=-\frac{\partial \omega}{\partial \mathbf{x}}
\end{gathered}
$$

where $\omega(K, \mathbf{x})$ is frequency and $\mathbf{K}$ is the wave number vector. Equation ( $2 a$ ) describes rays, which are paths traced by wave packets at group velocity. The change in the wave number vector with time is described by $(2 b)$. As waves are advected by a steady current, the frequency measured by a stationary observer remains constant

$$
\omega=\mathbf{K} \cdot\left(\mathbf{C}_{0}+\mathbf{U}\right)=\omega_{0}+\mathbf{K} \cdot \mathbf{U}
$$




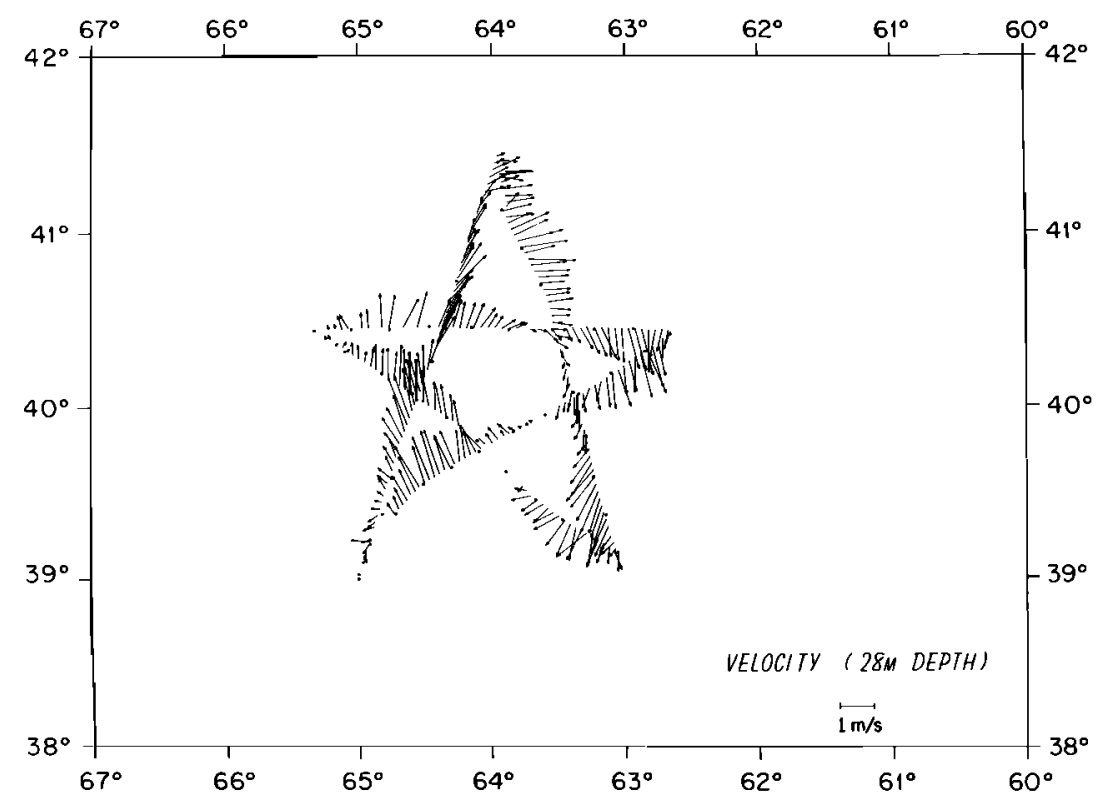

Fig. 1. Velocity field of surface current of a warm ring (5- to 28-m depth interval, 10-min average) measured by an acoustic Doppler current meter which records a continuous current profile while the ship is underway (September 14-16, 1981, after Joyce et al. [1983].

where $\mathbf{U}(\mathbf{x})$ is current velocity, $\mathbf{C}_{\mathbf{0}}$ is phase velocity with respect to the moving medium, and $\omega_{0}$ is frequency with respect to the moving medium. (Here, the subscript 0 refers to a frame of reference embedded in the moving medium.) The quantity $\omega_{0}$ is given by

$$
\omega_{0}=(g K)^{1 / 2}
$$

where $g$ is the acceleration of gravity. Therefore (in tensor notation)

$$
\frac{d x_{i}}{d t}=\frac{\partial \omega_{0}}{\partial K_{i}}+U_{i}=C g_{0_{1}}+U_{i}
$$

where $C_{g 0}$ is group velocity with respect to the moving medium. Also,

$$
\frac{d K_{\mathrm{t}}}{d t}=-K_{j} \frac{\partial U_{j}}{\partial x_{i}}
$$

describes changes in the wave number vector along the ray. Ray trajectories can be calculated by simultaneous integration of the following two equations:

$$
\begin{gathered}
x_{i}=\int\left(C g_{0,}+U_{i}\right) d t \\
K_{i}=-\int K_{j} \frac{\partial U_{j}}{\partial x_{i}} d t
\end{gathered}
$$

In summary, two points of note for waves propagating through a steady current are (1) in a homogeneous medium, frequency remains constant along a ray, as observed from a stationary frame, and (2) at any point on the ray, the wave number vector is not necessarily parallel to the ray.

Wave rays are most useful in examining wave propagation through nonuniform currents when the energy between adjacent rays can be considered constant as the distance between the ray paths changes. In deep water with waves of small and moderate amplitude, where wave breaking is rare, this assumption holds unless wave-current interaction by way of radiation stress [Longuet-Higgins and Stewart, 1961] is a factor. For currents in rings, Teague [1974] has shown that radiation stress is not significant if the phase velocity of the waves being refracted is much greater than the velocity of the maximum current in the ring.

\section{Current Field of a Warm Ring}

Measurements of surface currents in warm rings are scarce. Kitano [1975] reported a set of geomagnetic electrokinetograph (GEK) measurements, showing a maximum speed of $1 \mathrm{~m} \mathrm{~s}^{-1}$ for a Kuroshio warm ring of 100 nautical mile mean diameter. Andrews and Skully-Power [1976] reported a figure of $1.8 \mathrm{~m} \mathrm{~s}^{-1}$ for a $250-\mathrm{km}$-diameter ring off the coast of East Australia, using geostrophic calculations from hydrographic data. Saunders [1971] reported a maximum speed of $0.75 \mathrm{~m} \mathrm{~s}^{-1}$ for a $250-\mathrm{km}$-diameter Gulf Stream ring, measured by moored current meters when the ring passed through the array. Cheney [1976] reported a maximum velocity of $1.39 \mathrm{~m} \mathrm{~s}^{-1}$ for surface currents of a $160-\mathrm{km}$-diameter Gulf Stream ring, calculated from ship drift data.

A detailed set of measurements of surface currents of a warm core ring was reported by Joyce et al. [1983]. Using an acoustic Doppler current meter which operates continuously while the ship is underway, a profile of surface current $(10 \mathrm{~min}$ average over a depth interval of 5-28 m) was recorded for a series of transects tracing out the shape of a five-pointed star centered on the ring (Figure 1). The same ring was sampled twice, the first time when it was clearly separated from the Gulf Stream, the second time 10 days later when it was contacting the Gulf Stream. When separated from the Gulf Stream, the current field was characterized by circular flow, with the radial profile of tangential velocity approximating a Gaussian curve. When contacting the Gulf Stream, however, circular symmetry was destroyed.

Although there are no current data available at the time of the Seasat overpass examined here (September 21), there are hydrographic, ship drift, and Seasat altimeter data for the same ring earlier in 1978. Using these data, Gaborski [1979] calculated maximum current speeds on the order of $1 \mathrm{~m} \mathrm{~s}^{-1}$. 


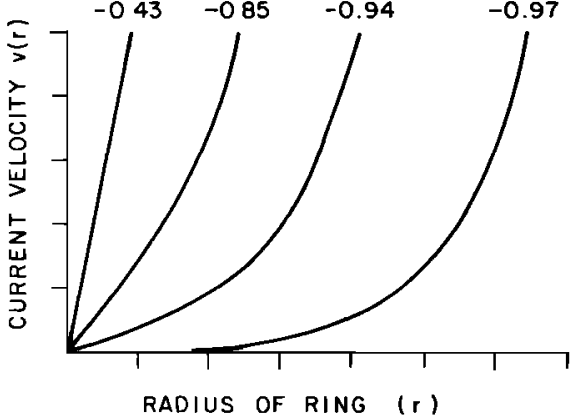

Fig. 2. Velocity profiles of warm core rings for various values of the parameter (Rossby number) after Flierl [1979].

\section{Mathematical Model of Refraction BY A WARM RING}

A mathematical model for the propagation of wave rays through the current field of a warm core ring was developed which performs stepwise integration of the ray equations ((5) and (6)), using a finite difference approximation. To run the model, initial wave conditions are specified, then the ray equations are integrated over time, yielding location coordinates and the wave number vector at each incremental time step.

\section{Flow Field}

The circular field of surface flow used to simulate the warm ring is specified by the radial profile of tangential velocity. The inner portion of the velocity profile, from the center to the point of maximum current velocity, is a power function of the form

$$
v(r)=C_{2} r^{n} \quad r \leq r_{j u}
$$

where $v(r)$ is current speed, $C_{2}$ is a scale factor for the amplitude of the velocity profile, $n$ is a constant chosen to produce

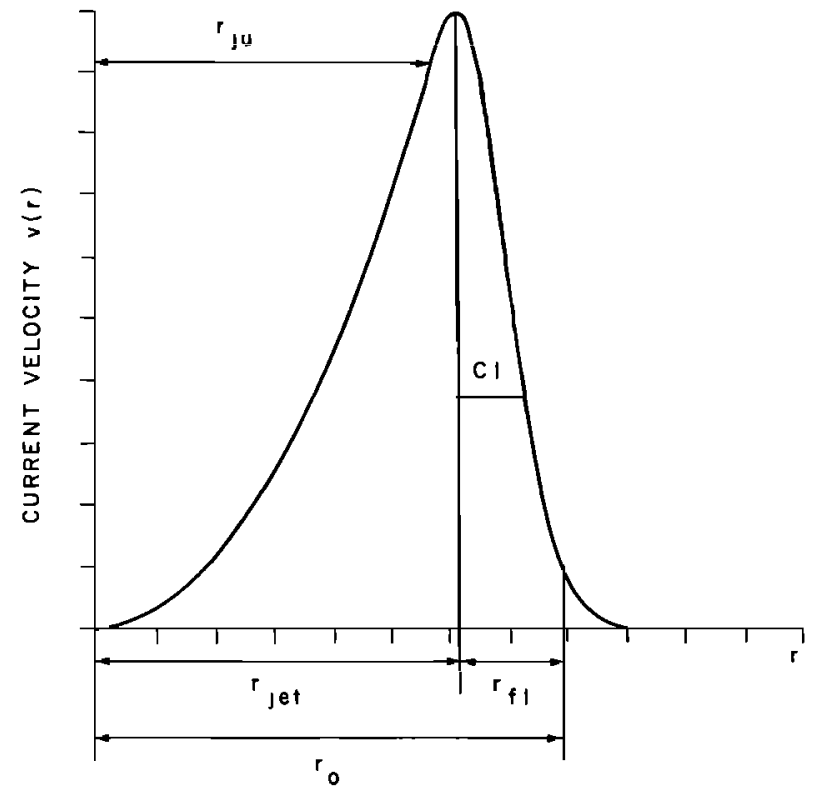

Fig. 3. Radial profile of tangential velocity used in the model to simulate the surface flow field of a warm core ring. Here $v(r)$ is current speed $(\mathrm{m} / \mathrm{s}), r$ is radial coordinate with respect to ring center $(\mathrm{km}), r_{j u}$ is radius of the junction point of the power and Gaussian functions $(\mathrm{km}), C_{1}$ is distance from point of maximum velocity at which velocity has decreased from maximum by a factor of $e(\mathrm{~km}), r_{\mathrm{jet}}$ is radius of current maximum $(\mathrm{km}), r_{f_{1}}$ is width of frictional layer $(\mathbf{k m})$, and $r_{0}$ is radius of ring, defined as point at which velocity has decreased from maximum value by a factor of $10(\mathrm{~km})$.
TABLE 1. Maximum Errors in Calculation of Wavelength and Propagation Direction due to Numerical Integration

\begin{tabular}{lcccc}
\hline $\begin{array}{c}\text { Current } \\
\text { Direction }\end{array}$ & $\begin{array}{c}\text { Distance of } \\
\text { Ray Travel, } \\
\text { km }\end{array}$ & $\begin{array}{c}\text { Location } \\
\text { Error, } \\
\text { km }\end{array}$ & $\begin{array}{c}\text { Propagation } \\
\text { Direction } \\
\text { Error, deg }\end{array}$ & $\begin{array}{c}\text { Wavelength } \\
\text { Error, \% }\end{array}$ \\
\hline Following & 60 & 0.175 & 0.90 & 1.2 \\
Opposing & 50 & 0.138 & 0.28 & 0.5 \\
\hline
\end{tabular}

At $30^{\circ}$ intervals of grid rotation, errors were tallied for incident angles of $10^{\circ}, 30^{\circ}$, and $50^{\circ}$ for a following current, and $10^{\circ}, 50^{\circ}$ and $90^{\circ}$ for an opposing current. Errors were calculated at a time interval of $16 \frac{2}{3} \mathrm{~min}$. Initial wavelength was $150 \mathrm{~m}$, and calculation time increment was $10 \mathrm{~s}$.

the desired velocity profile, and $r_{j u}$ is the radius of the junction point of the power and Gaussian functions, as described below. This function was selected as an approximation to the range of possible velocity profiles presented by Flierl [1979], illustrated in Figure 2.

Velocity profiles predicted by the Flierl model are discontinuous at the ring edge, due to the assumption of a twolayer fluid. In the model, this discontinuity was avoided by using a Gaussian curve to connect the power function (equations (8)) smoothly to the stationary region exterior to the ring. Smoothing the discontinuity represents the actual continuously stratified fluid. The Gaussian curve is given by

$$
v(r)=C_{2} \exp \left\{-\left[\left(r_{\mathrm{jel}}-r\right) / C_{1}\right]^{2}\right\} \quad r>r_{j u}
$$

where $C_{1}$ is the radial distance from the Gaussian peak at which $v=v_{\max } / e$. The outermost ring of the current field (where $r>r_{\text {jet }}$ ) will hereafter be called the frictional layer. An example of the composite velocity profile is shown in Figure 3.

\section{Verification of the Model}

For the special case of a linearly increasing, onedimensional shear current, the ray equations have been integrated analytically [Kenyon, 1971, equation 10]. This analytical solution was used as a standard for algorithm verification as well as for evaluation of errors attributable to numerical integration. Variables computed by the numerical model were subtracted from analytical results, yielding the error attributable to numerical integration (see Table 1 for maximum errors).

\section{Measuring the Wave Field FROM SAR IMAGERY}

Optical Fourier transform (OFT) techniques were used to measure wavelength and direction from 1-cm-square subimages. With the azimuthal scale of $1: 638,300$ on the image used, the subimages corresponded to oblongs on the sea surface with an azimuthal distance of $6.4 \mathrm{~km}$ on a side, and so contained about 50 waves of the nominal $120-\mathrm{m}$ length. The subimages were arranged in an alternating pattern in the area shown in Figure 7, and the OFT's themselves are shown, arranged in the same pattern, in Figure 4. The sampled subimages comprise about $14 \%$ of the total area of the image. In the OFT's, first-order maxima were estimated by choosing the distinct point of maximum optical density, where one existed, or by estimating the centroid of diffuse optical density maxima. To estimate the precision of these measurements, three subimages were chosen to represent various degrees of wave clarity, a subjective indicator of wave visibility. On each subimage, 10 measurements were made of wavelength and direction. For the data pooled from the three tests, standard 

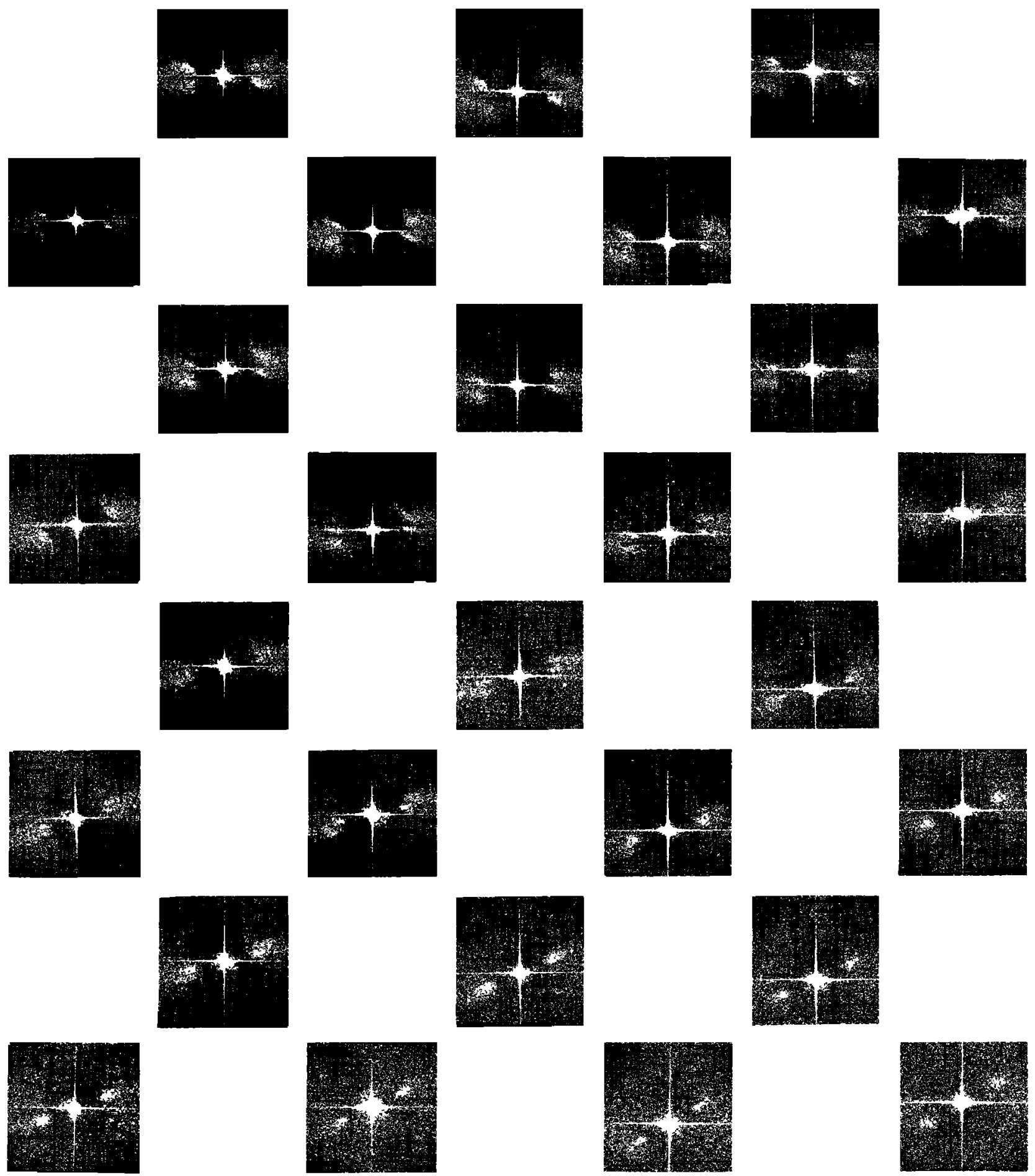

Fig. 4. Optical Fourier transforms taken from warm core ring image, orbit 1232. Top of page is oriented toward the northeast.

errors for direction and wavelength estimates were $1.0^{\circ}$ and $1.9 \mathrm{~m}$ respectively.

Measurements of ground range, wavelength, and propagation direction were corrected for slant range distortion in the following manner. All measurements were made initially as if the imagery were uniform in scale. Ground range was calculated using equation 5.1.1(6) of $W u$ et al. [1981]. After computing the local range scale, wavelength and direction were corrected by recalculating the range component of a vector proportional in length to wavelength and orthogonal to wave crests (hereafter referred to as an "orthogonal vector").

\section{Variability of Wave Clarity on SAR Imagery}

Wave clarity varied considerably in areas outside the ring on optically correlated imagery, which has a resolution of 40 $\mathrm{m}$ (as opposed to $25 \mathrm{~m}$ for digitally correlated imagery). (See Vesecky and Stewart [1982] for a discussion of the two correlation processes for SAR imagery and resulting resolution.) Immediately north and south of the ring, swell could not be resolved on OFT's. North of the ring, the image is dark, almost black in places. South of the ring, however, the tone is not distinctly different from that within the ring, but the texture becomes increasingly mottled. 




Fig. 5. "Typical" wave ray diagram produced by model. Shading applied to the hypothesis that in crossed seas, wave components from central rays have higher energy density than those from interfering rays. In unshaded areas the hypothesis is expected to hold while in shaded areas, the reverse is true. Circles correspond to $r_{\text {jet }}$ and $r_{0}$, the maximum current position and outside edge of the ring.

Vesecky and Stewart [1982] specify three requirements for resolution of ocean swell on SAR imagery.

1. Wavelength of sufficient magnitude to be resolved, a potential problem for waves traveling in the azimuth direction, where orbital motion degrades resolution. This did not appear to be the problem for this SAR swath, as waves were traveling within $30^{\circ}$ of range. Assuming that waves were indeed present in areas adjacent to the ring where they were not resolved, their wavelength would be roughly equal to that of other waves on the SAR swath, which were of sufficient wavelength to be resolved. If this assumption is correct, insufficient wavelength is not the cause of the gaps in wave resolution.

2. Significant wave height greater than approximately $1 \mathrm{~m}$.

3. Winds capable of producing wavelets of $30-\mathrm{cm}$ length, which are responsible for Bragg reflection of the radar beam.

According to Vesecky and Stewart [1982], dark areas on SAR imagery have been correlated to low wind speed. Such an area appears north of the ring, which suggests that low wind speed is inhibiting wave resolution. South of the ring, where the mottling disappears, waves gradually become visible. The mottling appears to inhibit wave resolution, and itself may be caused by winds.

\section{Model Results AND Discussion}

After initial debugging, the model was run through a series of tests designed to determine sensitivity to variables affecting the current velocity profile. From these tests, it became apparent that the refraction patterns varied between rays of extreme curvature, much as light rays would vary on refraction from a glass lens shaped in cross section like the velocity profile of the ring. In subsequent runs, rays were directed at points of the velocity profile corresponding to points of maximum and minimum curvature. The most conspicuous and consistent feature of the simulated wave fields (Figure 5) was a pattern of alternating zones of ray convergence and divergence located beyond the ring from left to right. (In this paper, ring locations are referred to from the viewpoint of the wave source.)

It was also evident that rays penetrating into the central region of the ring tended to pass through the ring without being refracted sharply. Rays incident upon the ring at grazing angles (such as rays $3,4,11$, and 12 of Figure 5 and rays 1 and 5 of Figure $6 a$ ), however, were refracted sharply to the left, crossing other relatively straight rays. After crossing, these rays formed an expanding "beam" of interfering rays. If the assumption is made that energy is conserved between rays, based on the large water depth compared to the wavelength and the large ratio of the wave phase speed to ring current maximum velocity, then energy of this beam decreases at a rate proportional to its angular divergence. Therefore in crossed seas the relative energy of the component from the beam of interfering waves should be less than that of other rays, which have generally not diverged as much. An exception to this rule is the shaded area of Figure 5, where the beam has not yet diverged sufficiently. The size of this shaded area decreases with increasing current velocity and decreasing incident wavelength.

The change in wavelength was examined for five rays of equal initial wavelength as they propagated through the simu- 


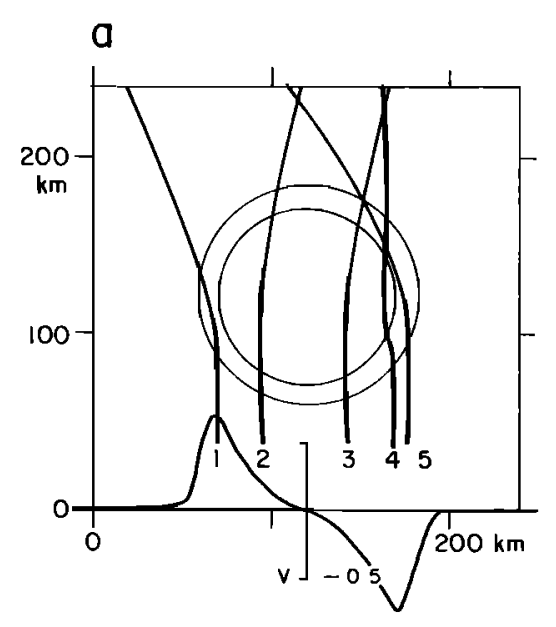

b
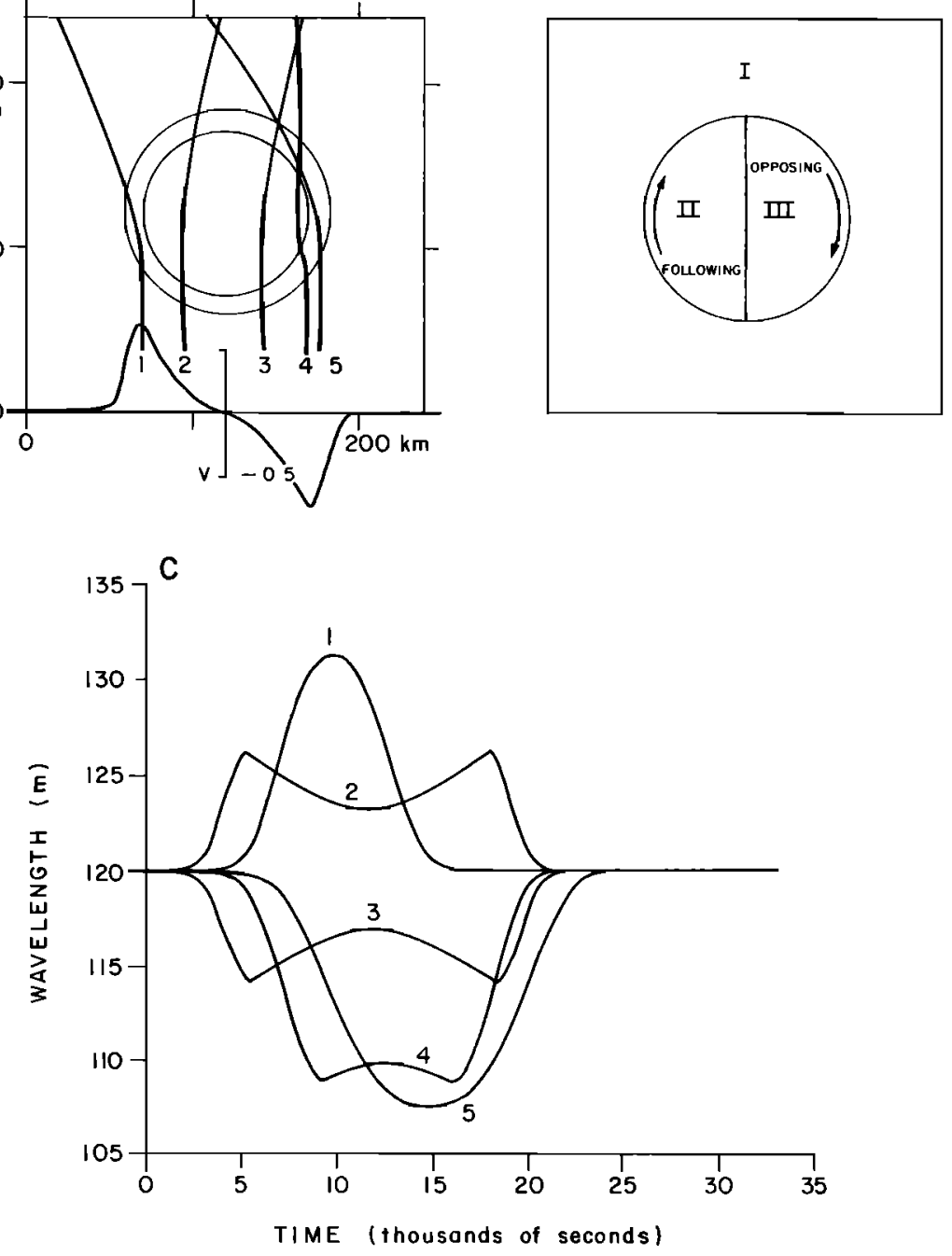

Fig. 6. (a) Five typical rays. (b) Regions of stationary water (I), following current (II), and opposing current (III). (c) Plot of wavelength versus time for rays of Figure $6 a$.

lated ring (Figure 6). The general pattern is as follows: After entering the following (opposing) current, the wavelength approaches a maximum (minimum) at the point of maximum current velocity, after which it approaches a local minimum (maximum) at the point nearest the ring center. As the ray exits the ring, wavelength increases (decreases) to a maximum (minimum) at the current velocity maximum and reverts to its initial wavelength outside the ring. Rays which do not penetrate inside the maximum current have only a single extremum in wavelength. Accordingly, an hypothesis, testable with the observed wave field, was formulated as follows. In region I of Figure $6 b$, where the water is stationary, the wavelength should be uniformly equal to that of the incident waves. In region II, where there is a component of current following the waves, the mean wavelength should be greater than that of region I. Conversely, in region III, where the current is opposing, mean wavelength should be less than that of region $I$.

It is interesting to note that the model predicts approximately equal wavelengths for the components of crossed seas (again assuming equality of initial wavelengths). For example, the difference in wavelengths at the point of intersection of rays 3 and 5 , and rays 4 and 5 of Figure $6 a$ is 1.4 and $1.6 \mathrm{~m}$, respectively. In both cases, the difference is unresolvable by the measurement technique.

Overall, there are three testable hypotheses applicable to any warm ring.

1. There are alternating zones of crossed seas and divergence behind the ring.

2. Assuming uniformity of incident wavelength, values of wavelength are as predicted by the model, and illustrated in Figure $6 c$.

3. In crossed seas, the relative energy density is greater for components of rays which passed through the central portion of the ring or missed the ring entirely, than for those comprising the beam of interfering rays. This hypothesis can be tested assuming that the OFT of a SAR image is approximately a two-dimensional variance density spectrum [Vesecky and Stewart, 1982]. Accordingly, the brightness of the firstorder maxima is proportional to the energy density of the wave. Therefore the relative energy density of each wave component of a bimodal OFT may be determined by ordering the brightness of first order maxima. (For this test, only monotonicity of brightness with energy density is required, not strict proportionality.) 


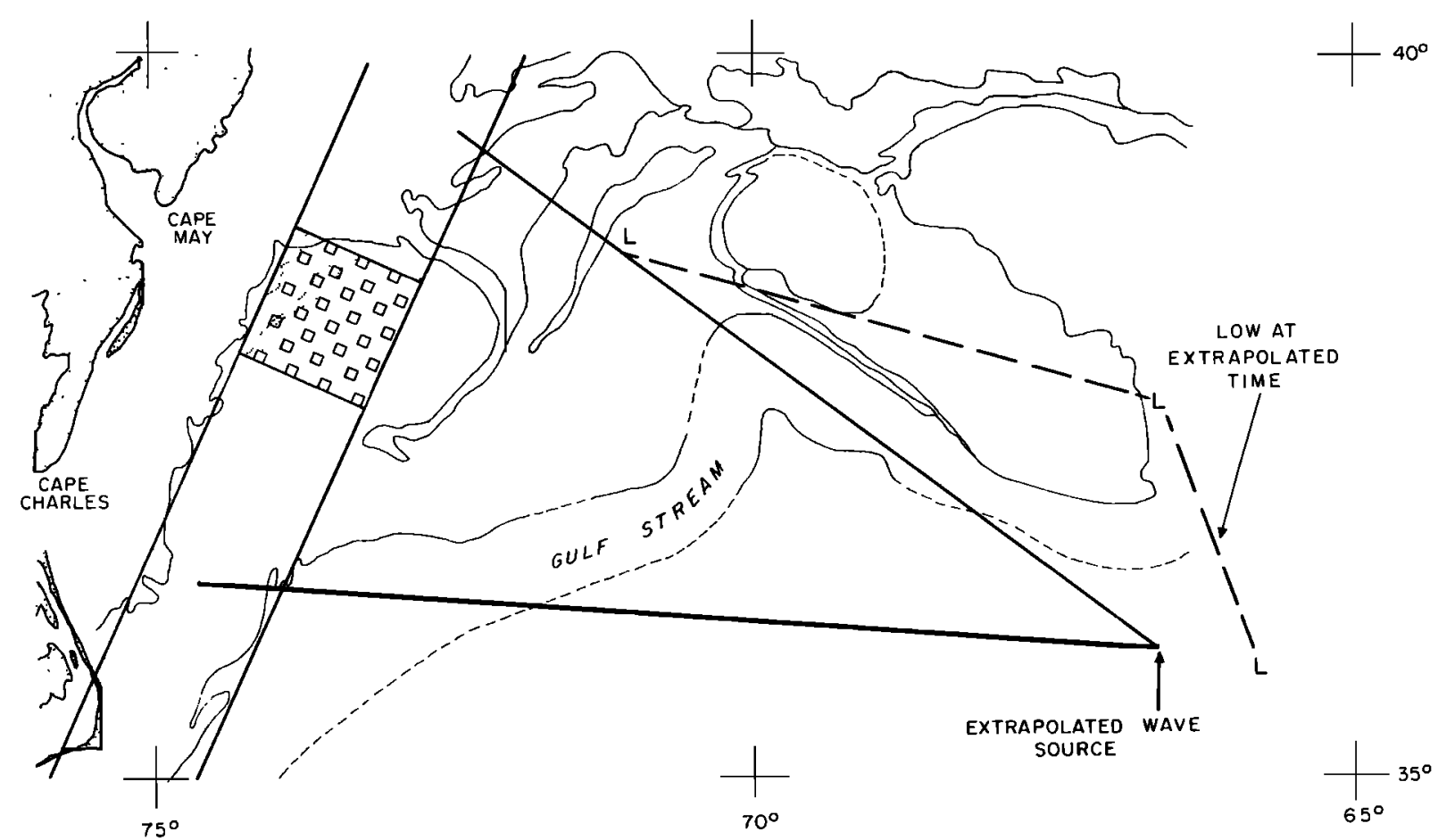

Fig. 7. Map of SAR swath showing extrapolated source of swell in relation to the Gulf Stream and a low-pressure cell. The Gulf Stream location is from a NAVOCEANO frontal analysis map, September 28 to October 4, 1978. Position of low is from a map of cyclone tracks [U.S. Environmental Data Service, 1978]. The Seasat orbit was September 20, 1978, 2300 EST. Waves of $120 \mathrm{~m}$ would take 25 hours to travel $620 \mathrm{~km}$ from the extrapolated source. The extrapolated time of generation was 1800 EST, September 19.

\section{COMParison OF OBSER VED AND Predicted Wave FieldS}

\section{Setting Initial Conditions for the Simulation}

Because of low wave clarity near the ring and the limits of the SAR swath, measurements of waves entering the ring could not be obtained to provide initial conditions. Measurements were therefore obtained for rays north and south of the ring, and the initial conditions for rays entering the ring were set by linear interpolation between measurable points. To examine the plausibility of this interpolation, an apparent source was located by extending orthogonals from the measured points to the point of intersection seaward from the measurements, and a plausible meteorological source was sought from meteorological data. In Figure 7, this apparent source is shown in relation to the Gulf Stream and a lowpressure system. The Gulf Stream location was taken from a NAVOCEANO Frontal Analysis map (September 28). The position of the low was taken from a map of cyclone tracks [U.S. Environmental Data Service, 1979]. Swell of 120-m wavelength would travel the distance of the apparent source (620 $\mathrm{km}$ ) in a time interval of 25 hours (at group velocity). At the extrapolated time of wave generation, the low was situated at the point indicted on the figure, making it a plausible source. The relative location suggests that the waves passed through the Gulf Stream before impinging on the ring. In view of the lack of data required to make detailed calculations of refraction by the Gulf Stream and the general uniformity of the wave field where it was clear outside the ring, refraction by the Gulf Stream was assumed to be uniform.

Ring radius, as measured both from thermal infrared (Heat Capacity Mapping Mission satellite, September 20, 1978) and SAR imagery (September 21, 1978, $0400 \mathrm{UT}$ ), was $65 \mathrm{~km}$.
There were no available data from which to determine the current velocity profile. Consequently, the three variables determining the shape of the profile ( $n$ of (8), $C_{1}$ of (9), and $C_{2}$ of (8) and (9)), were adjusted until a good fit, determined by visual comparison of the SAR-observed wave field to a series of simulations, was achieved. In the best fitting simulation, the maximum current velocity was $0.7 \mathrm{~m} \mathrm{~s}^{-1}$, and the frictional layer width was $15.1 \mathrm{~km}$. See Figure 8 for comparison of predicted and observed wave fields.

\section{Hypothesis Testing}

The simulated wave field was then compared in detail to the SAR-measured wave field. Three hypotheses were tested.

1. The hypothesis that the model predicts relative locations of convergence and divergence. A zone of divergence is present on the left side of the SAR image, as predicted by the model. A large region of crossed seas appears on the right side of the image both inside the ring and beyond. This area of crossed seas is larger than predicted, and the apparent source of the beam of interlering rays is "lower" in the ring than predicted. Also, the observed angular divergence of rays passing through the central portion of the ring was greater than predicted.

2. The hypothesis that the model correctly predicts wavelengths, assuming that the incident wavefield was uniform in wavelength. The imaged portion of the ring was divided into three regions corresponding to I, II, and III of Figure $6 b$. The division is shown in Figure 9. Of four specific tests, only one passed, i.e., mean wavelength in region II (following currents) was larger than that of region I (126.4 $\mathrm{m}$ versus $117.9 \mathrm{~m})$. The model prediction that wavelengths were uniform beyond the ring was not observed (standard deviation was $9.5 \mathrm{~m}$, which is much larger than the standard error of the mean of the 


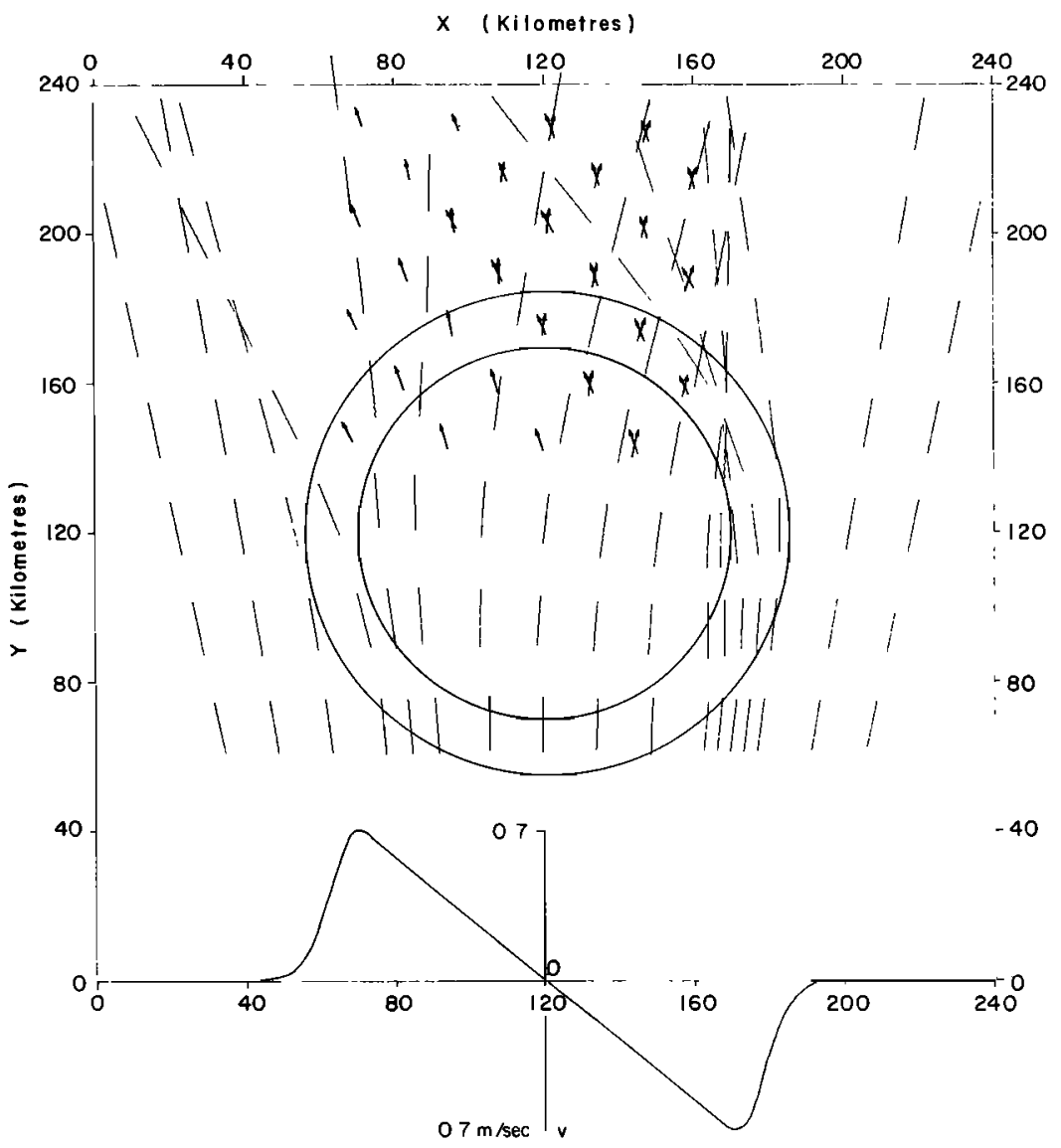

Fig. 8. Comparison of observed and predicted wave orthogonal directions. The observed SAR field is represented by short arrows, occupying a rectangular area in the top half of the figure. Predicted orthogonal directions are plotted along wave rays, the orthogonal direction differing most from the trend of the ray in regions of high transverse current shear. Circles correspond to $r_{\mathrm{jec}}$ and $r_{0}$, as defined in Figure 3.

measurement technique). Mean wavelength in region III (opposing currents) was approximately equal to that of region I $(115.4 \mathrm{~m}$ versus $117.9 \mathrm{~m})$, rather than less as predicted. Finally, the prediction of equal wavelengths in crossed seas was not observed. In most cases, the component from the interfering beam had a significantly smaller wavelength than other rays (Figure 9).

3. The hypothesis regarding relative wave energy in crossed seas. As predicted, the component in crossed seas from the interfering beam had less energy than other rays in 14 cases of 18 (Figure 9). In this instance, the area where the hypothesis does not apply (the shaded area of Figure 5) is relatively small, because of higher current velocity for this simulation.

\section{Discussion}

The digital image was from water of suflicient depth to rule out significant refraction by shoaling. Waves begin to refract from shoaling when depth becomes less than half the wavelength [U.S. Army Coastal Engineering Research Center, 1977]. There was one data extraction point located in water of transitional depth (i.e., less than half the wavelength). At this particular location, wave crests were oriented parallel to the local isobath, so refraction by shoaling was nil.

The model correctly predicted the relative location of convergence and divergence zones. There was, however, a larger area of crossed seas visible in the SAR image than predicted. Also, relative wavelength observed in crossed seas was smaller for components of the beam of interfering waves than for other components.

A plausible reason for smaller wavelengths in the beam of interfering waves is that the shorter wavelengths are refracted more than the longer wavelengths, the resulting dispersion selecting short waves in the measurement region. The sensitivity test of Figure 10 shows the (small) difference in refraction of $100 \mathrm{~m}$ waves versus $140 \mathrm{~m}$ waves, the extreme wavelengths of a group composed of a band of wavelengths of $120 \pm 20 \mathrm{~m}$ (roughly that observed). Comparing Figures $10 a$ and $10 b$, the difference in angular divergence of the interfering beam is $4^{\circ}$, the larger divergence corresponding to the smaller wavelength. Therefore dispersion is to a small extent responsible for (1) a larger area of crossed seas than predicted and for (2) smaller wavelengths at the left edge of the diverging beam. Dispersion alone, however, is not large enough to fully account for either the larger areas of crossed seas or the inequality of wavelengths in crossed seas observed in the SAR imagery.

Other hypotheses for larger areas of crossed seas than predicted by the model are (1) improper simulation of incident wave direction, (2) failure of linear wave theory to accurately describe refraction beyond ray crossings, and (3) oversimplified representation of the current field.

Generally, the tests of hypotheses regarding wavelengths failed. These tests were based on the assumption of a uniform incident wavelength. It is not clear whether this assumption or the model is inappropriate. There was, however, an area south of the ring where several measurements of wavelength were 
SAR ORTHOGONAL VECTORS

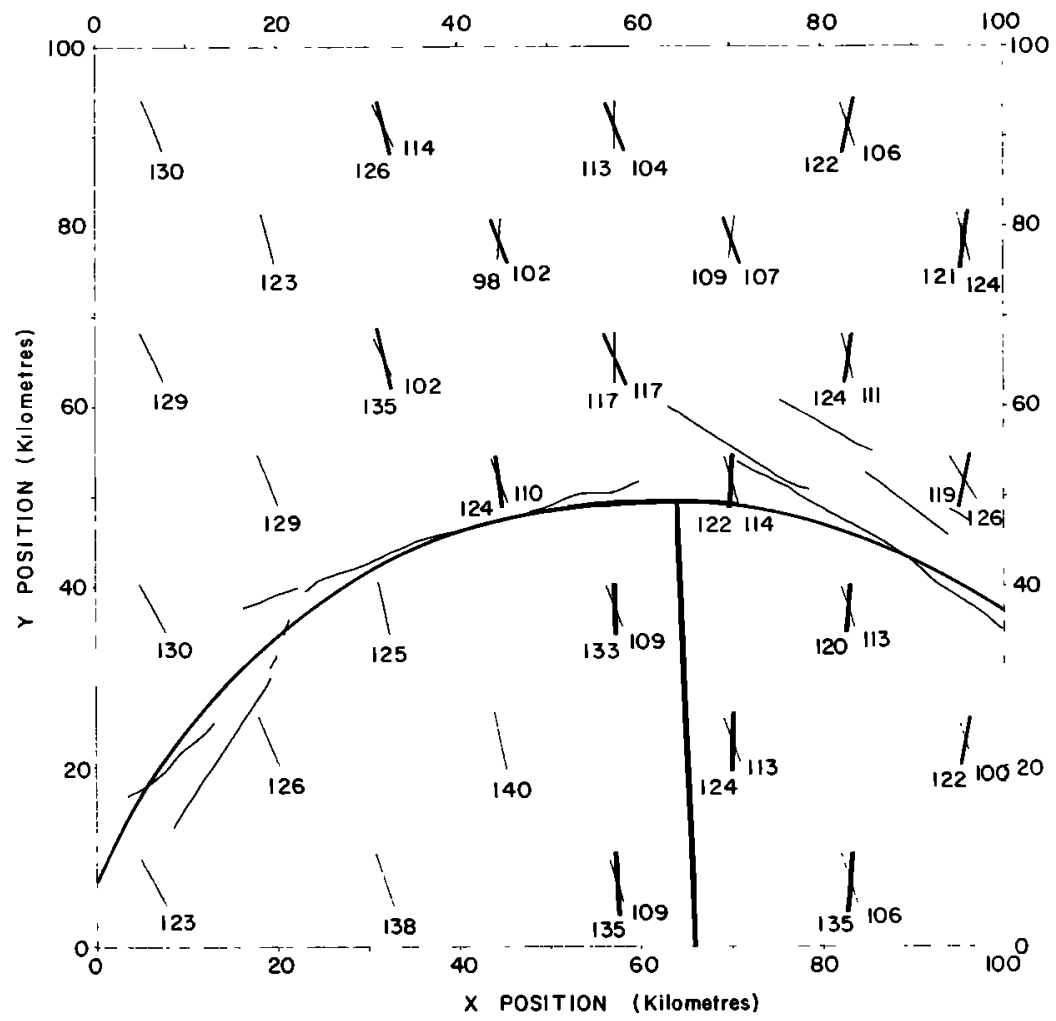

Fig. 9. Plot of orthogonal vectors for orbit $\mathbf{1 2 3 2}$ showing wavelengths (in meters), boundaries of zones described in Figure $6 b$, and arcuate linear features. Relative energy density of wave components in crossed seas is shown also. Wide lines indicate higher energy density.

made. Of eight measurements taken from an area roughly the same size as the ring, the standard deviation was $10 \%$. Part of this variance is due to poorer spatial resolution of optically correlated imagery, but it suggests that the assumption of uniform incident waves may be inappropriate.

If the model presented here is indeed representative of nature, the convergence of wave energy on each side of the ring should result in locally heavy seas. At times, the rays may be "focused" sufficiently to produce large pulses in the sea surface. Such waves, called "freak" ocean waves [Draper, 1964] or rogue waves, result from instantaneous superposition of many wave components. These waves appear without warning and are potentially catastrophic for surface vessels. The focusing of rays by currents increases the probability of such an event.

Ideally, further testing of this model would require numerous SAR overpasses with simultaneous measurements of currents.

\section{CONCLUSION}

Results suggest that the model provides a reasonable first approximation of observed wave refraction. The model accurately predicted the relative location of convergence and divergence zones as well as the relative energy density of components comprising crossed seas. The prediction of wavelengths, based on the assumption of uniform wavelength in the inci-



(A)

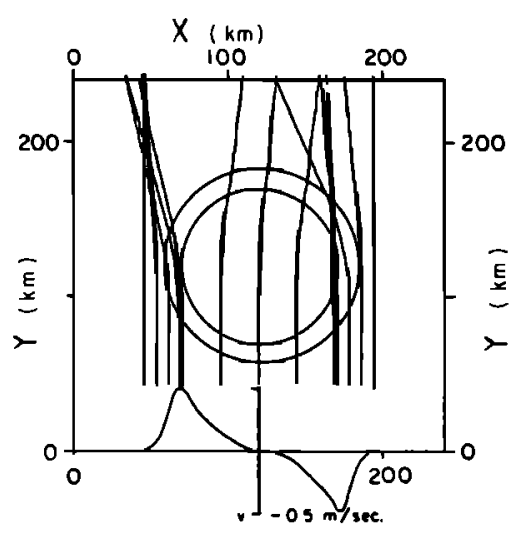

(B)

Fig. 10. Sensitivity test of refraction pattern for wavelengths of $(a) 100 \mathrm{~m}$ and $(b) 140 \mathrm{~m}$, approximate extreme values of observed wavelengths. 
dent wave field, was generally not observed. Beyond the ring, where wavelengths were predicted to be uniform, they were not. Within the left half of the ring (in the northern hemisphere, viewed from the direction of the waves) where there is a following current, wavelengths were greater than elsewhere, as predicted. However, on the right half of the ring, where currents are opposing, wavelengths were equal to initial values, rather than less as predicted. Finally, in crossed seas, wavelength was less for rays which had grazed the right edge of the ring, than for rays which had passed through the central portion of the ring. The model predicted nearly equal wavelengths in crossed seas.

Acknowledgments. The authors acknowledge the support of NOAA/NESDIS/CESL contract NA79 SAC 00775, under which the data analysis techniques were developed and tested. Support in preparation and publication is also gratefully acknowledged from the Virginia Institute of Marine Science. Particular help in manuscript and figure preparation was received from C. Gaskins, K. Stubblefield, and W. Jenkins. One of us (G.R.M.) acknowledges the help of the School of Marine Science, College of William and Mary, during work on a related Master's thesis. The SAR digital imagery was provided by $R$. C. Beal of Johns Hopkins University Applied Physics Laboratory. Finally, the reviewers gave helpful and supportive comments on the original version.

\section{REFERENCES}

Andrews, J. C., and P. Skully-Power, The structure of an East Australian Current anticyclonic eddy, J. Phys. Oceanogr., 7, 756-765, 1976.

Beal, R. C., P. S. DeLeonibus, and I. Katz (Eds.), Spaceborne Synthetic Aperture Radar for Oceanography, The Johns Hopkins University Press, Baltimore, Md., 1981.

Cheney, R. E, A census of rings in the Gulf Stream system, Tech. Note 3700-44-76, U.S. Naval Oceanogr. Office, Bay Saint Louis, Miss., 1976.

Draper, L., "Freak" ocean waves, Oceanus, $X, 12-15,1964$.

Flierl, G. R., A simple model for the structure of warm and cold core rings, J. Geophys. Res., 84, 781-785, 1979.

Gaborski, P. D., Observations of mesoscale circulation phenomena using SEASAT altimeter data and digital infrared imagery, Eos Trans. $A G U, 60,232,1979$.
Hayes, J. G., Ocean current wave interaction study, J. Geophys. Res., $85,5025-5031,1980$.

Johnson, J. W., The refraction of surface waves by currents, Eos Trans. $A G U, 28,867-874,1947$.

Joyce, T., M. Stalcup, and R. W. Schmitt, Aust. J. Mar. Freshwater Res., 34(4), 515-524, 1983.

Kenyon, K. E., Wave refraction by currents, Deep Sea Res., 18, 10231034, 1971.

Kitano, K., Some properties of the warm eddies generated in the confluence zone of the Kuroshio and Oyashio currents, J. Phys. Oceanogr., 5, 245-252, 1975.

LeBlond, P. H., and L. A. Mysak, Waves in the Ocean, $602 \mathrm{pp}$., Elsevier, New York, 1978.

Lichy, D. E., M. G. Mattie, and L. J. Mancini, Tracking of a warm water ring, in Spaceborne Synthetic Aperture Radar for Oceanography, edited by R. C. Beal, P. S. DeLeonibus, and I. Katz, pp. 171-182, Johns Hopkins University Press, Baltimore, Md., 1981.

Longuet-Higgins, M. S., and R. W. Stewart, The changes in amplitude of short gravity waves on steady non-uniform currents, $J$. Fluid Mech., 10, 529-549, 1961.

Saunders, P. M., Anticyclonic eddies formed from shoreward meanders of the Gulf Stream, Deep Sea Res., 18, 1207-1219, 1971.

Teague, W. J., Refraction of surface gravity waves in an eddy, Master's thesis, 66 pp. plus appendixes, Univ. of Miami, Rosensteil Sch. of Mar. and Atmos. Sci., Miami, Fla., Dec. 1974.

U.S. Army Coastal Engineering Research Center, Shoreline Protection Manual, vol. 1, U.S. Govt. Printing Office, Washington, D. C., 1977.

U.S. Environmental Data Service, Climatological Data, National Summary for September 1978, U.S. Govt. Printing Office, Washington, D. C., 1979 .

Vesecky, J. E., and R. H. Stewart, The observation of ocean surface phenomena using imagery from the SEASAT synthetic aperture radar: An assessment, J. Geophys. Res., 87, 3397-3430, 1982.

Wu, C., B. Barkan, B. Honeycutt, C. Leang, and S. Pang, An introduction to the interim digital SAR processor and the characteristics of the associated SEASAT imagery, 82 pp., Jet Propulsion Lab. Calif. Inst. of Technol., Pasadena, 1981.

G. R. Mapp, Computer Sciences Corporation, National Space Technology Laboratory, MS 39529.

J. C. Munday and C. S. Welch, Virginia Institute of Marine Science, School of Marine Science, College of William and Mary, Gloucester Point, VA 23062.

(Received March 3, 1984; accepted June 18, 1984.) 